\title{
Supporting Information: Photocurrent quantum yield in suspended carbon nanotube pn junctions
}

\author{
Lee Aspitarte, Daniel R. McCulley, Ethan D. Minot \\ Department of Physics, Oregon State University, Corvallis, OR, 97331
}

Corresponding author: ethan.minot@oregonstate.edu

\section{Electrical characteristics of split gate suspended CNT devices}

Figure S1 shows the conductance of a split-gate suspended CNT device when $V_{\mathrm{g} 1}=V_{\mathrm{g} 2}=V_{\mathrm{g}}$ and $V_{\text {sd }}=25 \mathrm{mV}$. In this configuration, the device behaves as a field-effect transistor (rather than a pn junction). The high conductance at negative gate voltage (p-type doping) contrasts the negligible conductance at positive gate voltage (n-type doping). The asymmetric behavior indicates that the Fermi level at the ends of the CNT is pinned by the metal contacts to an energy near the valance band edge. When negative gate voltages are applied, hole transport in unimpeded by the metal-semiconductor interface. When positive gate voltage is applied, electron transport is blocked by a large n-type Schottky barrier (see band bending diagrams in Fig. S1). For $V_{\mathrm{g}}>0$, the device conductance remains below the noise floor $(<10 \mathrm{pS})$. There is no detectable gate leakage current. 
(a)

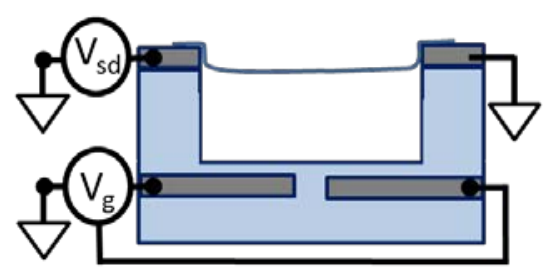

(b)

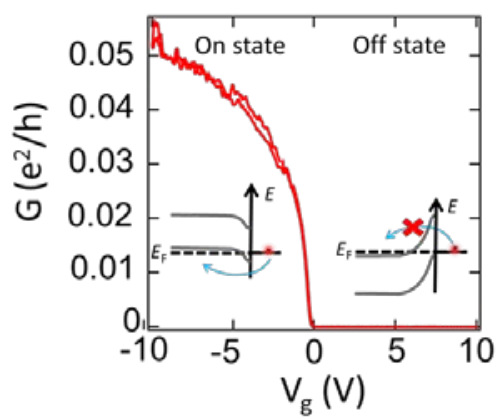

Fig. S1. a) Schematic and b) Conductance $G\left(V_{\mathrm{g}}\right)$ of a split-gate suspended CNT device operated as a field effect transistor.

Figure S2 shows $I-V_{\text {sd }}$ characteristics of a split-gate suspended device that is gated into a pn junction configuration. All measurements were made in the dark (no laser illumination). Changing the magnitude of the gate voltages $V_{\mathrm{g} 1}$ and $V_{\mathrm{g} 2}$ causes dramatic changes in the diode characteristics. Similar suspended CNT pn junction characteristics were previously reported by Liu et al. The cause of the dramatic changes in diode characteristic can be traced to the n-type Schottky barrier. ${ }^{1}$ 
(a)

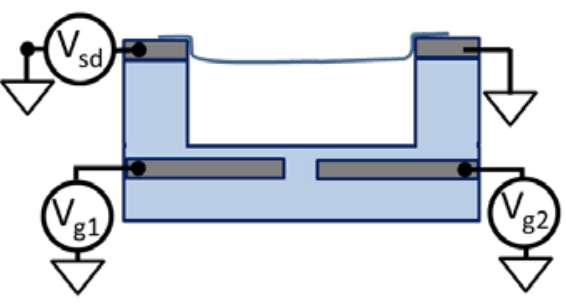

(b)

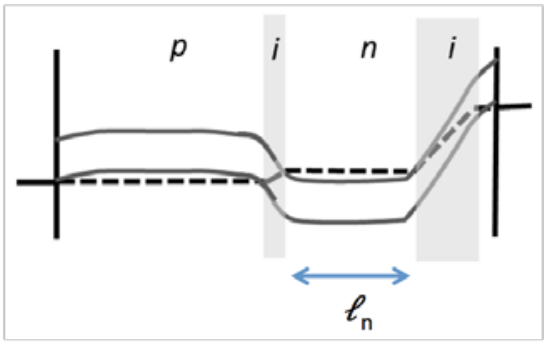

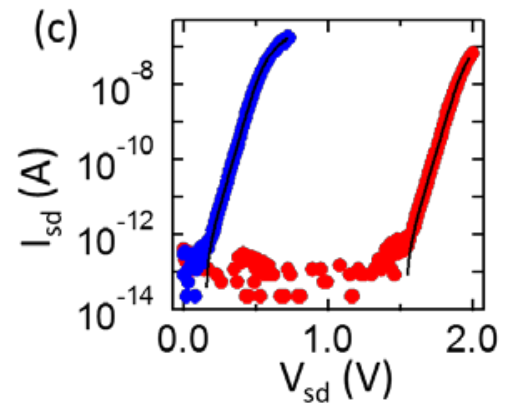

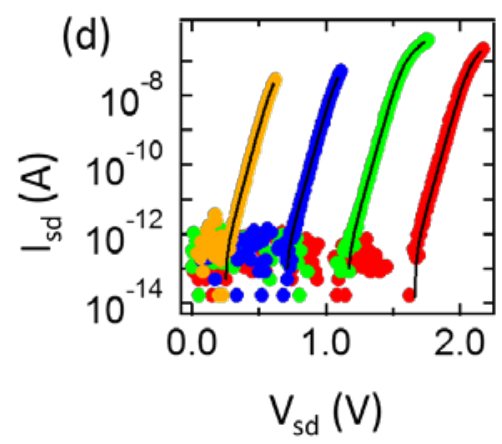

(e)

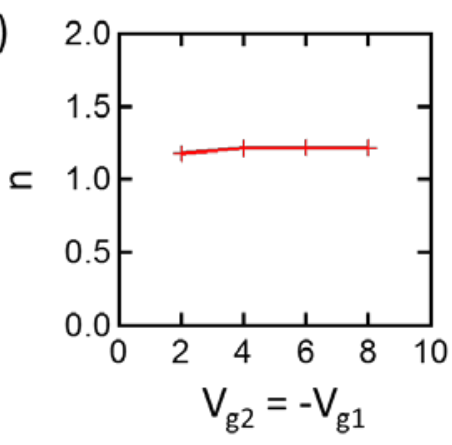

Figure S2. a) Device schematic. b) Band bending when the pn junction is in forward bias and the n-type Schottky barrier is in reverse bias. c) Current versus $V_{\text {sd }}$ data for device S1. Red dots correspond to symmetric n-type and p-type gating $\left(V_{\mathrm{g} 2}=-V_{\mathrm{g} 1}=8 \mathrm{~V}\right)$. Blue dots correspond to asymmetric gating $\left(V_{\mathrm{g} 2}=4 \mathrm{~V}, V_{\mathrm{g} 1}=-8 \mathrm{~V}\right)$. Equation $\mathrm{S} 1$ is used for curve fitting (black lines). Fit lines have ideality factor $n=1.29\left(V_{\mathrm{g} 2}=-V_{\mathrm{g} 1}=8 \mathrm{~V}\right)$ and $1.25\left(V_{\mathrm{g} 2}=4 \mathrm{~V}, V_{\mathrm{g} 1}=-8 \mathrm{~V}\right)$. d) Device S2. Lightly gated to heavily gated. Orange, blue, green and red correspond to $V_{\mathrm{g} 2}=-V_{\mathrm{g} 1}=2,4,6$, and $8 \mathrm{~V}$ respectively. Equation S2 is used for curve fitting (black lines). e) The ideality factors for the fitting curves in part $\mathrm{d}$.

With increasing positive $V_{\mathrm{sd}}$, the pn junction device reaches a threshold where an exponentially increasing current is observed with ideality factor near unity (Fig. S2c and d). This exponential regime is followed by a roll-off to a linearly increasing current. The exponential increase and roll-off can be fitted well to a model that combines a modified diode and a series resistor. The modified diode model is 


$$
I=I_{0}\left[\exp \left(\frac{V-V_{\text {shift }}}{n V_{\mathrm{Th}}}\right)-1\right]
$$

where $I_{0}$ is the reverse-bias leakage current, $V$ is the voltage drop across the pn junction, $n \geq 1$ is the dimensionless ideality factor, $V_{\text {Th }}$ is the thermal voltage, $26 \mathrm{mV}$, and $V_{\text {shift }}$ is a threshold shift.

The $V_{\text {shift }}$ term is not present in a standard diode model. We use $V_{\text {shift }}$ here to capture the effect of the Schottky diode that opposes the pn diode in the suspended CNT devices. When $V_{\text {sd }}>0$, the Schottky diode is in reverse bias and the pn junction is in forward bias (see Fig S2b). When 0 $<V_{\text {sd }}<V_{\text {shift}}$, current is limited by the rate at which n-type carriers can leak through the reverse biased n-type Schottky barrier. This current is below the noise floor of our experiments, but can be seen in the measurements of Liu et al. who observed larger currents in the bias window $0<$ $V_{\text {sd }}<V_{\text {shift. }}{ }^{1}$

When $V_{\text {sd }}>V_{\text {shift, }}$, the length of the n-type region, $\ell_{n}$, is sufficiently short that minority carriers can traverse the n-type region and reach the right-side electrode (see Fig S2b). This condition is met when $\ell_{\mathrm{n}}$ is less than the minority carrier diffusion length in the n-type region. When $V_{\text {sd }}>$ $V_{\text {shift, }}$ current is no longer limited by the n-type Schottky barrier. Instead, current is determined by thermally-activated hole transport over the pn junction.

Combining the diode model, eq $\mathrm{S} 1$, with a series resistance, $R$, gives ${ }^{2}$

$$
I=I_{0}\left[\frac{n V_{\mathrm{Th}}}{I_{0} R} W\left(\frac{I_{0} R}{n V_{\mathrm{Th}}} e^{\frac{V_{\mathrm{sd}}-V_{\mathrm{sh} \text { ift }}+I_{0} R}{n V_{\mathrm{Th}}}}\right)-1\right] .
$$

where $W$ is the Lambert $\mathrm{W}$-function. Fitting curves based on eq. S2 are shown in Figure S1. $I_{0}=$ $300 \mathrm{fA}$ is determined from high-sensitivity measurements of the reverse bias leakage current. $R \sim$ $1 \mathrm{M} \Omega$ is determined from the linear $I-V_{\text {sd }}$ regime $(I>1 \mathrm{nA})$. The ideality factor, $n$, is close to unity for all $I$ - $V_{\text {sd }}$ curves $(1.2<n<1.3)$. 
The gating configuration causes dramatic changes in $V_{\text {shift. }}$ Liu et al. explain this phenomenon by considering the effect of $V_{\mathrm{g} 1}$ and $V_{\mathrm{g} 2}$ on the length $\ell_{\mathrm{n}}$. Asymmetrically gating the device with a larger positive gate $\left(\left|V_{\mathrm{g} 2}\right|>\left|\mathrm{V}_{\mathrm{g} 1}\right|\right.$, Figure S2c) increases $\ell_{\mathrm{n}}$. This results in a larger $V_{\text {shift. We }}$ also find that increasing $\left|V_{\mathrm{g} 1}\right|$ and $\left|V_{\mathrm{g} 2}\right|$ symmetrically changes $V_{\text {shift }}$ (Fig S2d). In general, a larger $\left|\mathrm{V}_{\mathrm{g} 2}\right|$ causes a larger electric field within the n-type Schottky barrier, $\varepsilon_{\mathrm{Sch}}$. Large $\varepsilon_{\mathrm{Sch}}$ makes $\ell_{\mathrm{n}}$ less sensitive to $V_{\text {sd. }}$.

\section{Gate capacitance}

We performed Coulomb blockade spectroscopy on one of our devices. The device was operated as a single-electron transistor by cooling to low temperature and applying the same voltage, $V_{\mathrm{g}}$, to both split gates. In this regime, gate capacitance and total capacitance can be accurately determined (Figure S3). ${ }^{3}$

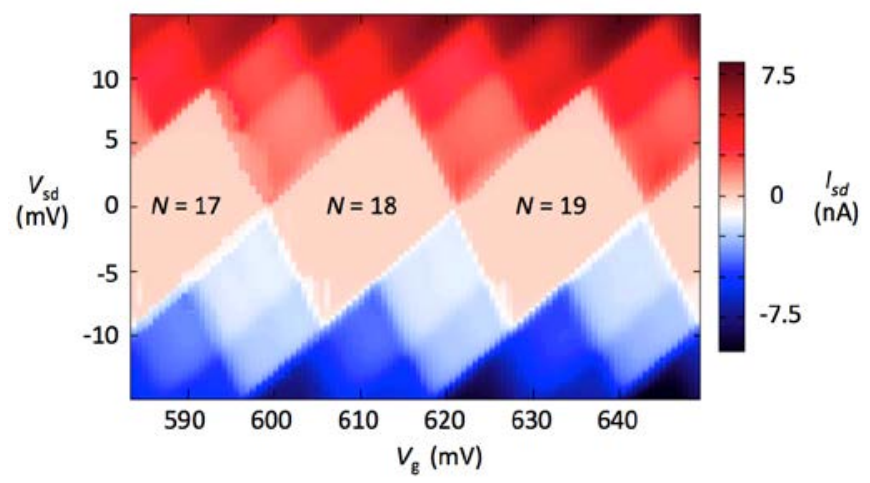

Figure S3. Coulomb blockade measurements at $30 \mathrm{mK}$. (Collaboration with Gary Steele at TU Delft). Labels show the number of holes occupying the dot. The height of the blockade diamonds is $9 \mathrm{mV}$. The width of the diamonds is $22 \mathrm{mV}$. 
The width of the Coulomb diamonds depends on the gate capacitance, $C_{\mathrm{g}}$, the total capacitance, $C$, and the energy level spacing $\Delta E$ :

$$
\Delta V_{\mathrm{g}}(N \rightarrow N+1)=\frac{e}{C_{\mathrm{g}}}+\left(\frac{C}{C_{\mathrm{g}}}\right) \frac{\Delta E}{e} .
$$

From Figure S3 we find $\Delta V_{\mathrm{g}}(N \rightarrow N+1)=22 \mathrm{mV}$. For a $2 \mu \mathrm{m}$-long CNT, $\Delta E<1 \mathrm{meV}$. We conclude that $C_{\mathrm{g}} \sim 7 \mathrm{aF}$.

The gate capacitance can be used to estimate the charge density per unit length, $\lambda$, for different gating configurations. For example, if $\left|V_{\mathrm{g}}\right|=10 \mathrm{~V}$, then

$$
|\lambda|=\frac{7 \mathrm{aF}}{2 \mu \mathrm{m}}(10 \mathrm{~V})=220 \frac{e}{\mu \mathrm{m}},
$$

where $e$ is the elemental charge. If the device is operated as a pn junction, the split gate voltages are equal and opposite $\left(V_{\mathrm{g} 1}=-V_{\mathrm{g} 2}\right)$. The left gate has some coupling to the right side of the CNT and vice versa. Therefore, $|\lambda|$ will be slightly less when the device is operated in the pn junction configuration.

(1) Liu, C. H.; Wu, C. C.; Zhong, Z. Nano Lett. 2011, 11 (4), 1782-1785.

(2) Buchs, G.; Barkelid, M.; Bagiante, S.; Steele, G. a.; Zwiller, V. J. Appl. Phys. 2011, 110 (7), 074308.

(3) Kouwenhoven, L. P.; Marcus, C. M.; Mceuen, P. L.; Tarucha, S.; Robert, M. In Mesoscopic electron transport; Springer Netherlands, 1997; pp 105-214. 